\title{
Study on Etiological Factors, Clinical Pattern and Comorbidities in Cerebral Palsy Children of North Bihar Region
}

\author{
Anil Kumar ${ }^{1}$, Kripa Nath Mishra², Sanjay Kumar Nirala ${ }^{3}$, Prashant Gaurav${ }^{4}$, Randhir Kumar Mishra ${ }^{5}$, Jaimala ${ }^{6}$
}

${ }^{1}$ Department of Paediatrics, Darbhanga Medical College and Hospital, Darbhanga, Bihar, India. ${ }^{2}$ Department of Paediatrics, Darbhanga Medical College and Hospital, Darbhanga, Bihar, India. ${ }^{3}$ Department of Paediatrics, Darbhanga Medical College and Hospital, Darbhanga, Bihar, India. ${ }^{4}$ Department of Paediatrics, Darbhanga Medical College and Hospital, Darbhanga, Bihar, India. ${ }^{5}$ Department of Paediatrics, Darbhanga Medical College and Hospital, Darbhanga, Bihar, India. ${ }^{6}$ Department of Paediatrics, Darbhanga Medical College and Hospital, Darbhanga, Bihar, India.

\section{ABSTRACT}

\section{BACKGROUND}

Cerebral palsy (CP) is a form chronic motor disability in children. It is a nonprogressive disorder of posture and movement, often associated with epilepsy and abnormalities of speech, vision and intellect. It results from a defect or lesion of growing brain before or during birth or in postnatal period. The damage to brain is permanent and cannot be cured but the consequences can be minimized. Cerebral palsy (CP) is a disorder of development in which abnormalities of motor function are the main characteristic features. Severity varies from mild to severe. Cerebral palsy is a very challenging disability for parents and professionals. Cerebral palsy is a heterogeneous group of disorders caused by intrapartum asphyxia and exposure to maternal infection such as chorioamnionitis, sepsis, urinary tract infection, and prematurity. The incidence of Cerebral Palsy is 2 to 2.5 per 1000 live births. During the past twenty years, there have been increases in the incidence and prevalence of $\mathrm{CP}$ that may be associated to enhanced records of cases, advances in neonatal health care and other factors.

\section{METHODS}

This cross-sectional study was conducted in the Department of Paediatrics, Darbhanga Medical College \& Hospital, Laheriasarai, Darbhanga, Bihar, from October 2018 to September 2019. A total of 100 Cerebral Palsy cases coming from various districts to inpatient and outpatient department of hospital were selected.

\section{RESULTS}

In this study a total $100 \mathrm{CP}$ children up to 12 years of age was included. $60 \%$ were boys and $40 \%$ were girls. The causes of CP included birth asphyxia (47\%), prematurity (22\%), pyogenic meningitis (8\%), genetic $(7 \%)$, neonatal sepsis $(6 \%)$, intracranial bleed $(5 \%)$, and idiopathic $(5 \%)$. Among these cases, spastic type (65\%), ataxic $(15 \%)$, dyskinetic $(10 \%)$ and mixed $(10 \%)$ varieties were found. Among spastic, quadriplegic subtype was seen in $69 \%$, diplegia in $23 \%$, and hemiplegia in $8 \%$. Comorbidities associated with CP patients were speech problem $(80 \%)$, pain $(75 \%)$, cognitive disability $(50 \%)$, hip displacement $(30 \%)$, seizure (25\%), behavioural disorders (25\%), sleep disturbances $(20 \%)$, visual impairment (19\%) and hearing impairment (4\%).

\section{CONCLUSIONS}

Perinatal asphyxia is a leading and preventable cause of cerebral palsy. Spastic type was the most common type and in spastic type, quadriplegic subtype was most common. Mental retardation, speech problems, pain, cognitive disability, hip displacement and seizure were the major co-morbidities in children with cerebral palsy. Early diagnosis and appropriate management are important in such children to limit morbidity.

\section{KEY WORDS}

Cerebral Palsy (CP), Perinatal Asphyxia, Quadriplegic, Spasticity
Corresponding Author: Dr. Kripa Nath Mishra, Department of Paediatrics, DMCH, Laheriasarai, Darbhanga-846003, Bihar, India.

E-mail: kripanathmishra1@gmail.com

DOI: $10.14260 /$ jemds/2020/294

Financial or Other Competing Interests: None.

How to Cite This Article:

Kumar A, Mishra KN, Nirala SK, et al. Study on etiological factors, clinical pattern and comorbidities in cerebral palsy children of North Bihar region. J. Evolution Med. Dent. Sci. 2020;9(16):1349-1353, DOI: $10.14260 /$ jemds/2020/294

Submission 04-12-2019,

Peer Review 28-03-2020,

Acceptance 03-04-2020,

Published 20-04-2020.

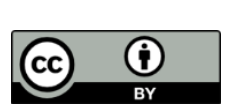




\section{BACKGROUND}

Cerebral Palsy (CP) is a form chronic motor disability in children worldwide with the greatest burden in developing countries. ${ }^{1}$ Cerebral palsy is a non-progressive disorder of posture and movement often associated with epilepsy and abnormalities of speech, vision and intellect. It results from a defect or lesion of growing brain before or during or in postnatal period. The damage to the brain is permanent and cannot be cured but the consequences can be minimized. The motor disorders are frequently accompanied by sensory impairment, behavioural problem, intellectual impairment, epilepsy, speech disorders, hearing problems, ocular defects, failure to thrive, growth failure, skeletal deformities and joint contractures. CP is the most common reason of crippling in children. Boys have more incidence and more severe course than girls (male: female ratio 1.4:1). The damage to developing brain is permanent and cannot be cured but sequelae can be minimized. Residual impairments or sequelae hinder with the skill to function in daily life, decreases quality of life that produce even greater activity limitation than the motor impairments. These are the hallmarks of CP.1,2

$\mathrm{CP}$ is caused by developmental brain anomaly, intrauterine hypo perfusion (IUGR), perinatal asphyxia, prematurity associated with white matter injury, hypoglycaemia, bilirubin encephalopathy, congenital intrauterine infection (TORCH), sepsis, meningitis in neonatal period, encephalitis in early infancy that produce a common group of neurological disorder. Upper age of limit of brain insult is $2-3$ years. Only $10 \%$ of CP cases can be recognized to neonatal asphyxia. Mostly, brain insult occurs during perinatal period and search for specific causes becomes very difficult. Cerebral anoxia, often accompanied by intraventricular and subependymal haemorrhage followed by physical birth trauma to the brain leads to CP. Antenatal causes of $\mathrm{CP}$ are prematurity, low birth weight, maternal epilepsy, untreated hypothyroidism, TORCH infection, eclampsia, multiple pregnancy, insufficiency of placenta, drug abuse, smoking, Rh incompatibility and bleeding during pregnancy. Perinatal risk factors are prolonged and obstructed labour, premature rupture of amniotic membranes, abruption of placenta etc. Postnatal causes are CNS infection (meningitis, encephalitis), hypoxia, seizure, coagulopathy, neonatal hyperbilirubinemia and head trauma. Infertility treatments are also related with greater chances of CP. About $2 \%$ of $\mathrm{CP}$ cases are inherited as autosomal recessive with both parents being carriers. In milder $\mathrm{CP}$, brain grossly appears normal but is underweight and has only sparse subcortical white matter and sparse nerve fibres. The finding in severe CP includes extensive cerebral atrophy, cavity formation in subcortical white matter, atrophy of basal ganglia and porencephaly.

Typically, children who are going to have CP show neurodevelopmental delay in infancy. This is due to delayed disappearance of primitive reflexes and late appearance of postural responses. In a child with $\mathrm{CP}$, the primitive reflexes persist, and advanced postural reaction/maturational reflexes do not appear. An abnormal movement pattern emerges as the child grows. Most common clinical features are delayed motor \& mental milestones, abnormal reflexes, abnormal muscle tone (hypotonic/hypertonic), abnormal movement/ seizure, hemiplegia, growth retardation, gait abnormalities, skeletal deformities and joint contractures. Problem with posture, balance, coordination, walking, speech, hearing, vision, swallowing, bowel and bladder etc are also associated with CP child.

$\mathrm{CP}$ is a form of chronic motor disability. The worldwide prevalence of CP ranges from 1.5 to more than 4 per 1000 live births or children of defined age group. ${ }^{3}$ In India, it is approximately around 3 cases per 1000 live births, however the actual data may be more than expected data. Since mild cases are likely to be missed in surveys, the prevalence of $\mathrm{CP}$ may well be higher than this estimate. In India approximately, there are 25 lakhs CP children. The prevalence and pattern of Cerebral Palsy varies between different geographical areas, perhaps because of different etiological factors.

The category of cerebral palsy depends on the site of brain involved. When the cerebral cortex or corticospinal fibres are involved, spastic cerebral palsy is seen, when the basal ganglia are affected, cerebral palsy with extrapyramidal features such as athetoid or dystonic features is seen. When cerebellum is affected, ataxic cerebral palsy is seen. In preterm babies, the cortex is not developed in the periventricular region, hence the leg fibres that cross in the pyramid tract are affected. This result in infantile diplegia.

Cerebral Palsy is classified physiologically as spastic, dyskinetic, ataxic, and mixed type. Spastic palsy may be quadriplegic, diplegic and hemiplegic while dyskinetic palsy may be choreoathetoid or dystonic. Spastic quadriplegia is the most common and most severe form of CP because of obvious motor impairment of all extremities and the high association with intellectual disability and seizures. Spastic diplegia is bilateral spasticity of the legs that is greater than in the arms and is linked to prematurity. Child of spastic diplegia reveals spasticity in the legs with brisk reflexes, ankle clonus and a bilateral Babinski sign.

The major aim of treatment in CP is to achieve maximum possible functional ability and skill in keeping with the child's development age. This is mainly achieved through physiotherapy (cornerstone of CP management), surgical correction, and occupational therapy. Early intervention programs involve identification of neonates who are at high risk of developing developmental delay and providing infant stimulation and caregiver education. Early intervention may retard or minimize the central nervous lesion causing the clinical picture of $\mathrm{CP}$ and thus prevent or minimize neuromotor delay.

$\mathrm{CP}$ is frequently associated with a broad range of developmental problem, a multidisciplinary advanced approach is most supportive in the evaluation and management of such children. Complete assessment of a child with CP, hence, should include an evaluation of related deficits such as microcephaly, vision problem, speech (communication), hearing, sensory profile, motor evaluation, epilepsy, malnutrition and cognitive functioning. $\mathrm{CP}$ is a chronic permanent condition with significant impact on affected individuals. Physical finding are persistence of neonatal reflexes, increased tone, fisting with cortical thumb, scissoring of legs, toe-walking, abnormal posture and gait, abnormal movement and hyperreflexia. Early diagnosis and comprehensive management with a multidisciplinary advanced approach involving neurodevelopmental paediatrician, neurologist, orthopaedic surgeon, speech and 
language therapist, physiotherapist, occupational therapist, rehabilitation specialists, social worker and educators are required for acceptable management of a child of CP. 4

Role of Paediatricians are early recognition \& early referral, management of nutrition related problems \& vaccination, Anti-epileptics \& control of seizure, control of excessive salivation, control of hyperactivity, relief of dystonia, management of GERD and sleep problems. Parental counselling, social awareness, Counselling for the cerebral palsied children and vocational support have important role in rehabilitation for $\mathrm{CP}$ child. Voluntary organizations such as the Spastic society of India, launched in 1972, and Indian family of CP, launched in 1993, to serve the interests of the CP affected children, need to be encouraged.

Better obstetric, perinatal and neonatal care prevents birth trauma, birth asphyxia and kernicterus. Logically, it should cut down the incidence of CP. However, it has recently been suggested that future developments targeted at enhancing perinatal care may have only marginal effect on the prevalence of $\mathrm{CP}$ as has been the case in prosperous countries. It may be worthwhile to direct $\mathrm{CP}$ related research to the area of development biology as well. Early and prompt detection of $\mathrm{CP}$ cases and adequate planning for management may help indirectly by reducing residual neurological, psychological and other handicaps and making child's life comfortable.

\section{Objectives}

- To assess the etiological factors and clinical pattern of Cerebral Palsy.

- To find out the prevalence of related disabilities or handicaps in Cerebral palsy.

\section{METHODS}

The present cross-sectional study was done between the children attending to the inpatient and outpatient Department of Paediatrics, Darbhanga Medical College and Hospital, Laheriasarai over duration of one year from October 2018 to September 2019 and, 100 Children presenting with features of CP were selected. Informed consent were taken from parents of all children enrolled in the study.

\section{Inclusion Criteria}

Children presenting with non-progressive neurodevelopment delay up to 12 years of age were enrolled in study.

\section{Exclusion Criteria}

Children presenting with non-central motor deficits and progressive neurological disorder were excluded from study.

All the medical records of child previously existing with the parents were analysed to search out the probable causes and evaluation of different comorbidities. Detailed history and physical examination were done using a systematically designed proforma to search out the related disability in children. Information were also noted from the parents as regards whether the children have had a proper vision and hearing evaluation, neuroimaging. Epilepsy in a child was diagnosed from history and EEG examination. Vision defect was assessed by history, clinical examination and fundoscopy. Hearing defect was assessed by history, clinical test and BERA. Information regarding availed physiotherapy, rehabilitation, and speech therapy services or attended any particular school were collected. Modified Swedish classification were used to categorize CP children. 4,5 Approval of the Institutional ethical committee was taken prior to conduct of this study.

\section{Statistical Analysis}

All data was collected and entered in Microsoft Excel and interpreted using Statistical Package for Social Sciences (SPSS) version 17. Descriptive statistics was used primarily. Categorical data were expressed in proportions and percentages.

\section{RESULTS}

A total 100 children of cerebral palsy were taken for this study of which $60 \%$ were boys and $40 \%$ were girls attending to inpatient and outpatient department of hospital from various districts. Different age group of CP patient and from different area were selected of which $<2$ yrs. (40\%), 2-4 year (35\%), 4-6 year (13\%), 6-12 year (12\%). The most common etiological factor are perinatal asphyxia (47\%), prematurity (22\%), pyogenic meningitis $(8 \%)$, genetic $(7 \%)$, neonatal sepsis (6\%), intracranial bleed (5\%), idiopathic (5\%). [table 1].

\begin{tabular}{|cc|}
\hline Factors & Number of Patients Affected, Percentage \\
Asphyxia & $47(47 \%)$ \\
Prematurity & $22(22 \%)$ \\
Pyogenic meningitis & $8(8 \%)$ \\
Genetic & $7(7 \%)$ \\
Neonatal sepsis & $6(6 \%)$ \\
Intracranial bleed & $5(5 \%)$ \\
Idiopathic & $5(5 \%)$ \\
\hline & Table 1. Etiological Factors \\
\hline
\end{tabular}

Patients of CP presented to hospital with a variety of problems like seizures (48\%), acute pulmonary infection (16\%), acute gastrointestinal tract infection (14\%) and genitourinary infection (6\%). Rest $17 \%$ of children had been brought to the hospital with delayed milestone as main complaints. In present study types of Cerebral Palsy patients was spastic type (65\%), Ataxic (15\%), Dyskinetic (10\%), and Mixed (10\%). In spastic, quadriplegic subtype was seen in $69 \%$, diplegia in $23 \%$, and hemiplegia in $8 \%$ [figure 1 and 2].

The comorbidities associated with $\mathrm{CP}$ patients were mental retardation $(90 \%)$, speech problem $(80 \%)$, pain (75\%), cognitive disability (50\%), hip displacement (30\%), seizure (25\%), behavioural disorders (25\%), sleep disturbances $(20 \%)$, visual impairment (19\%), and hearing impairment (4\%).

Formal visual assessment was made in $48 \mathrm{CP}$ patient, among them 30 patients had normal vision, 9 patients had refractive error, 7 patients were having strabismus and 2 patients were blind. Spectacles were used by only three $\mathrm{CP}$ patients. 

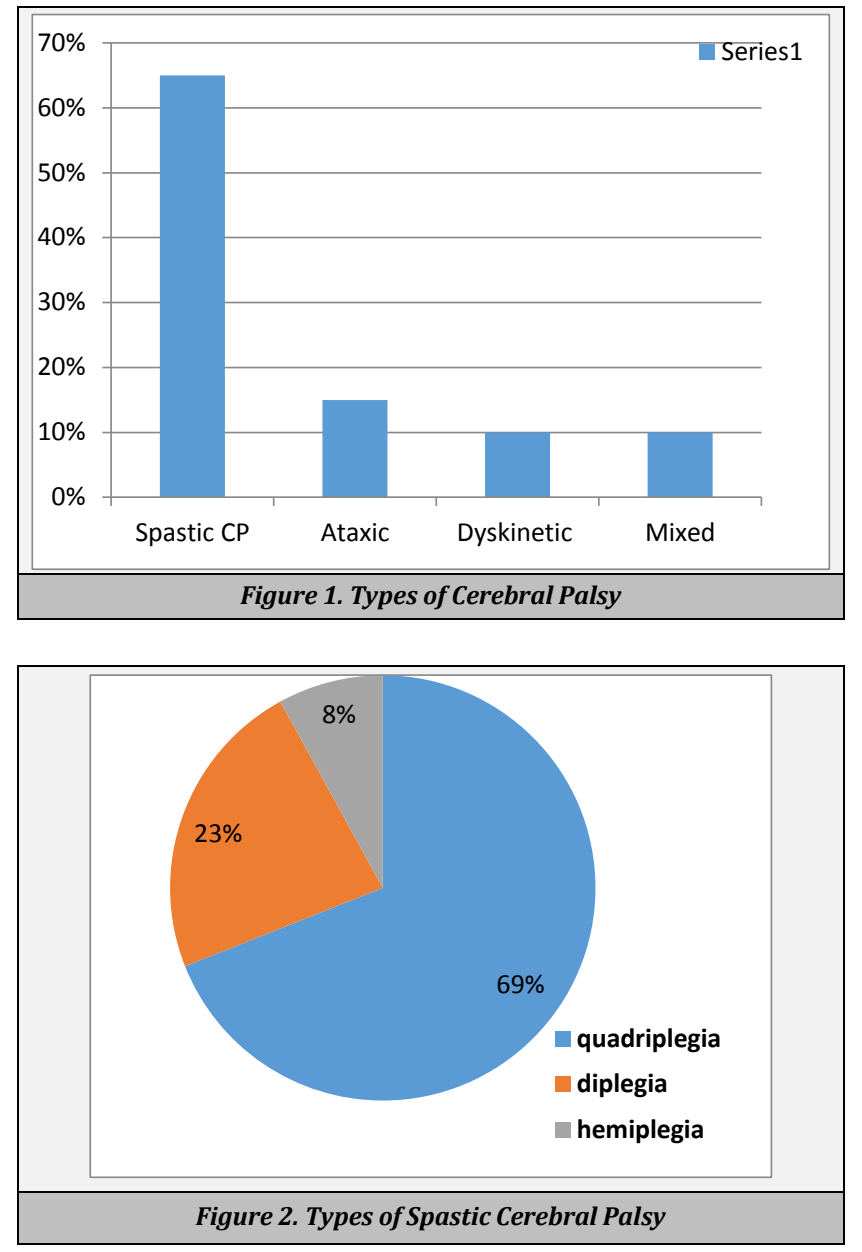

\section{DISCUSSION}

Different areas have different incidence and pattern of CP possibly due to variable risk factors. Different classification for study, used by different area are also responsible for different incidence \& pattern of CP. There is considerable decline in the prevalence and severity of CP subtypes and related disability in European countries, most probably due to advancements in obstetric, perinatal and postnatal care. $\mathrm{CP}$ prevalence in Jammu is $2.27 \%{ }^{6}$. CP prevalence in Norway, decline from 2.62 per 1000 live births in 1999 to 1.89 in $2010 .^{7}$ Overall occurrence of CP per 1000 live births decrease considerably from 3.3 in 1981 to 2.3 in $1990{ }^{8}$ in Slovenian children.

Both male and female are affected by CP; though, girls are relatively less affected by CP. In this present study, 60 children were boys and 40 children were girls, with a proportion of 1.5:1. Male child predominance has been found in various studies completed by Tatavarti et al.,9 Johnson ${ }^{10}$ in Europe, Laisram et al., ${ }^{11}$ and Das et al. ${ }^{12}$ in India.

Children of CP are brought to the tertiary care hospitals at an early age with either different health issues or concern mainly for development delay. In the present study, $40 \%$ of the children were $<2$ years while $35 \%$ were in the range of 2 and 4 years. A study of 100 children having $\mathrm{CP}$ at a tertiary care hospital by Gowda et al. conclude that age of CP child at the first diagnosis was $<1$ year in $28 \%$ and $1-4$ years in $65 \%{ }^{13}$ In the current study, spastic CP children were $65 \%$, of which
$69 \%$ were quadriplegic subtype and $23 \%$ were diplegic subtype. The epidemiological study of CP done by Odding et al. conclude that it is more common in underprivileged socioeconomic groups, with the majority having the spastic type of which diplegic subtype is the least. ${ }^{14}$ A study from North India done by Singhi et al. in 2002, observed quadriplegic spastic CP in $61 \%$ and diplegic CP in $20 \%$ of the total CP children. ${ }^{15}$ Singhi et al. in another study done in 2013, found $51.5 \%$ spastic quadriplegia and $34.5 \%$ diplegia. 16 A study Das et al. revealed $43 \%$ spastic quadriplegia and $12 \%$ spastic diplegia. Similarly, Gowda et al. reported $71 \%$ spastic quadriplegia and $16 \%$ spastic diplegia. Therefore, our results of present study are steady with all these Indian studies.

Perinatal risk factor such as birth asphyxia has been important role in CP in underdeveloped countries. Birth asphyxia is responsible for $47 \%$ of CP patients in our present study. Our outcome is in consistency with studies done by Singhi et al. who found $45.3 \%$ cases and in a different study $51.98 \%$ associated with birth asphyxia. In our study, other etiological factors noted are prematurity $(22 \%)$, pyogenic meningitis (8\%), genetic (7\%), neonatal sepsis (6\%), intracranial bleed (5\%), idiopathic (5\%). A study Singhi et al. observed, neonatal sepsis in $30.6 \%$ cases and neonatal hyperbilirubinemia in $35.14 \%$ cases. In another study of Singhi et al. reported neonatal septicemia $14.6 \%$ and neonatal hyperbilirubinemia $21.6 \% .^{15,16}$

The proper auditory evaluation had been done in only $30 \%$ of CP children, out of which 5 children were having moderate to severe loss of hearing (16.6\%). In current study, just $48 \%$ had undergone proper visual checkup, out of which 18 children (37.5\%) were having visual abnormalities. A study of Dass et al. had also revealed speech defect $(76 \%)$, visual problems $(23 \%)$, and auditory defects (18\%). Singhi et al. found, speech problems (83.7\%), visual defect $(46.7 \%)$, and auditory defect (13.9\%). Hence Our results are steady with these authors. ${ }^{12,15} \mathrm{~A}$ study of Sasmal et al. in 140 patients of $\mathrm{CP}$, also revealed an overall rate of ocular defect as $42.1 \%$. These were strabismus (36.4\%), myopia $(12.9 \%)$, hypermetropia $(8.6 \%)$, astigmatism $(3.6 \%)$, optic atrophy $(2.1 \%)$, and nystagmus $(2.1 \%)$. Cortical visual impairment was seen in $20.7 \% .{ }^{17}$

In our country, clinical pattern of CP patient differs from clinical pattern of western nations due to difference in perinatal and postnatal morbidities, for example, birth asphyxia, CNS infection, hypoglycaemia and kernicterus. Moreover, the proportion of CP children who have undergone ocular and auditory check-up and undergoing treatment is very little. Early diagnosis and start of suitable management should be provided to lower the incidence of Cerebral Palsy and related comorbidities in the future. It is recommended that rehabilitation centre should be establish for $\mathrm{CP}$ children at all government medical college so that parents of $\mathrm{CP}$ can be made sensitive of the illness, handicaps, prognosis and management.

\section{CONCLUSIONS}

It was observed in present study that perinatal factors were the most important risk factors in Cerebral Palsy (CP). We 
suppose that CP prevalence can successfully be minimized by improving the quality of monitoring obstetric care, regular checkup and by improving quality of delivery rooms, delivery teams and neonatal intensive care units. In addition, the effect of early maternity cannot be underestimated; hence increasing the community awareness concerning early marriage is of particular importance.

The study also highlights the various problems relating to cerebral palsy which must be detected at the earliest to aid in timely and proper management of patients. Lastly, even though $\mathrm{CP}$ is non curable illness, rehabilitation, special learning, and psycho-social support may considerably mitigate the problems and may offer reintegration of the child into the community.

\section{REFERENCES}

[1] Rosenbaum P, Paneth N, Leviton A, et al. The definition and classification of cerebral palsy. Dev Med Child Neurol 2007;49(s109):1-44.

[2] Morris C. Definition and classification of cerebral palsy: a historical perspective. Dev Med Child Neurol Suppl 2007;109:3-7.

[3] CDC Data and Statistics for Cerebral Palsy. Available from: https://www.cdc.gov/ncbddd/cp/data.html

[4] Sankar C, Mundkur N. Cerebral palsy-definition, classification, etiology and early diagnosis. Indian J Pediatr 2005;72(10):865-8.

[5] Khandaker G, Sheedy HS, Islam J, et al. Bangladesh cerebral palsy register (BCPR): a pilot study to develop a national cerebral palsy (CP) register with surveillance of children for CP. BMC Neurol 2015;15:173.

[6] Raina SK, Razdan S, Nanda R. Prevalence of cerebral palsy in children $<10$ years of age in R.S. Pura town of Jammu and Kashmir. J Trop Pediatr 2011;57(4):293-5.
[7] Hollung SJ, Vik T, Lydersen S. Decreasing prevalence and severity of cerebral palsy in Norway among children born 1999 to 2010 concomitant with improvements in perinatal health. Eur J Paediatr Neurol 2018;22(5):814-21.

[8] Kavcic A, Perat MV. Prevalence of cerebral palsy in Slovenia: birth years 1981 to 1990. Dev Med Child Neurol 1998;40(7):459-63.

[9] Tatavarti SR, Garimella RR, Subbalakshmi TD. Male sex preponderance in cerebral palsy. Int J Orthop Sci 2018;4(3):200-2.

[10] Johnson A. Prevalence and characteristics of children with cerebral palsy in Europe. Dev Med Child Neurol 2002;44(9):633-40.

[11] Laisram N, Srivastava VK, Srivastava RK. Cerebral palsy an etiological study. Indian J Pediatr 1992;59(6):723-8.

[12] Das N, Beboruah G, Das I. Study on the clinical profile of patients with cerebral palsy. IOSR J Dent Med Sci 2016;15(7):54-8.

[13] Gowda VK, Kumar A, Shivappa SK, et al. Clinical profile, predisposing factors, and associated co-morbidities of children with cerebral palsy in South India. J Pediatr Neurosci 2015;10(2):108-13.

[14] Odding E, Roebroeck ME, Stam HJ. The epidemiology of cerebral palsy: incidence, impairments and risk factors. Disabil Rehabil 2006;28(4):183-91.

[15] Singhi PD, Ray M, Suri G. Clinical spectrum of cerebral palsy in North India--an analysis of 1,000 cases. J Trop Pediatr 2002;48(3):162-6.

[16] Singhi P, Saini AG. Changes in the clinical spectrum of cerebral palsy over two decades in North India--an analysis of 1212 cases. J Trop Pediatr 2013;59(6):43440.

[17] Sasmal NK, Maiti P, Mandal R, et al. Ocular manifestations in children with cerebral palsy. J Indian Med Assoc 2011;109(5):318-23. 